\title{
Uma Estratégia Baseada em Difusão de Informação para Determinação de Conteúdos Relevantes e Usuários Influentes em Redes Sociais.
}

\author{
Hérico Valiati ${ }^{1}$ \\ Arlei Silva ${ }^{2}$ \\ Sara Guimarães ${ }^{1}$ \\ Wagner Meira Jr. ${ }^{1}$
}

Resumo: Redes sociais têm desempenhado um papel muito importante como um meio para a disseminação de informações e ideias, e exercício da influência de alguns usuários sobre outros. O problema alvo deste trabalho é determinar quais usuários são influentes e quais os conteúdos relevantes, ou seja, ordenar tanto grupos de usuários quanto o conteúdo por eles disseminado. Propomos uma nova técnica que se baseia em uma definição intuitiva e circular de relevância e influência. Nós descrevemos a técnica proposta em detalhes, assim como uma implementação eficiente para a mesma. A fim de validá-la, nós utilizamos técnicas de recomendação de usuários e conteúdo. Nós utilizamos duas bases de dados reais do Twitter e os resultados obtidos mostram que a técnica proposta apresenta ganhos de $37 \%$ quando comparada a um método de filtragem colaborativa. Além disso, tanto os usuários influentes quanto os conteúdos relevantes identificados pela nossa técnica são qualitativamente superiores. A principal contribuição desse trabalho é um estudo de caso no qual aplicamos a técnica proposta em um cenário real da Web. As análises comprovam que o nosso método consegue identificar usuários influentes e pode ser facilmente adotada em diversos cenários de aplicação.

\footnotetext{
${ }^{1}$ Departamento de Ciência da Computação - Universidade Federal de Minas Gerais

\{herico, sara, meira@dcc.ufmg.br\}

${ }^{2}$ Computer Science Department - University of California, Santa Barbara

arlei@cs.ucsb.edu
} 
Uma Estratégia Baseada em Difusão de Informação para Determinação de Conteúdos Relevantes e Usuários Influentes em Redes Sociais.

\begin{abstract}
Social networks are an increasingly important media for disseminating information, ideas, and users influencing other users. The main target of this work is to determine which users are influential and to identify relevant content, that is, rank user groups and content spread by them. We propose a novel technique that is based on an intuitive and circular definition of relevance and influence. We describe the proposed technique in detail, as well as its efficient implementation. In order to validate it, we considered the task of recommending users and content. We used two real data sets extracted from Twitter and our results show that our technique outperforms by $37 \%$ a collaborative filtering strategy, while both influential users and relevant content are qualitatively better. The main contribution of this work is a case study, where we apply the proposed technique in a real Web scenario. Analysis confirms that our proposal is able to identify influent users and may be easily adopted in a variety of application scenarios.
\end{abstract}

\title{
1 Introdução
}

Redes sociais têm desempenhado um papel cada vez mais fundamental como meio para a disseminação de informação, idéias e um lugar onde seus membros exercem influência uns sobre os outros. E isso ocorre mesmo além das redes, tendo em vista a crescente convergência que pode ser observada entre as várias mídias. Em suma, redes sociais têm se mostrado um importante mecanismo para atingir grandes parcelas da população, influenciando a opinião pública, a adoção de inovações, a publicidade de novos produtos ou marcas. Entretanto, identificar as características do conteúdo relevante, assim como dos usuários que os tornam populares ou impactantes é uma questão de pesquisa que vem sendo investigada sob várias perspectivas [20, 11]. Por exemplo, o problema de otimizar a disseminação de conteúdo e explorá-lo para fins de marketing já foi provado como NP-Difícil, assim como alguns de seus variantes [16]. Vários autores também se dedicaram a identificar e caracterizar as redes de difusão de informação [7, 21].

O problema alvo deste artigo é determinar tanto usuários influentes quanto conteúdo relevante, ou seja, ordenar tanto grupos de usuários quanto o conteúdo por eles disseminado. O conceito de influência é discuto em [2, 1, 9]. Nesse trabalho, consideramos a definição de influência como a capacidade de um usuário de afetar o comportamento de outros usuários [9]. Já um conteúdo relevante é aquele que vai de encontro às necessidades de informação de um determinado usuário, e é um conceito muito utilizado em recuperação de informação [3]. Há vários desafios ao realizar essas tarefas: (i) a rede de disseminação de informação é pouco observável, dificultando a detecção exata do processo de disseminação e, portanto, da relevância do conteúdo e da influência dos usuários; (ii) quando é possível observar essa difusão e identificar os principais fatores que a explicam, em geral, tratamos de eventos de sucesso, de alto impacto, que são raros e cujas características podem não se 
generalizar para outros tipos de influência e/ou relevância de interesse, como por exemplo eventos que se limitam a grupos ou contextos específicos; (iii) o comportamento dos usuários muda ao longo do tempo, e mais uma vez de forma pouco previsível e observável. Nesse caso, temos que levar em conta essa evolução e sermos capazes de lidar com ela.

Neste artigo propomos uma nova técnica para ordenar usuários e conteúdo, de acordo com a sua influência sobre outros usuários e com sua relevância, respectivamente. Essa nova técnica se baseia em uma definição intuitiva e circular de relevância e influência, ou seja, usuários influentes tendem a disseminar conteúdo relevante e conteúdo relevante é em geral disseminado por usuários influentes. É interessante notar que não nos concentramos em um grupo restrito e pequeno de conteúdos, mas em todo o tráfego disseminado por uma rede social. Essa definição foi modelada como uma extensão do problema de PageRank [5] e implementada eficientemente, uma vez que não requer a obtenção da rede de seguidores e determina as ordenações através de uma estratégia randomizada. A técnica proposta foi avaliada no contexto de dois cenários amplamente discutidos na rede social Twitter: política

e automóveis. É também interessante notar que a nossa metodologia de avaliação é baseada em recomendação, que é uma forma indireta, mas que se mostrou efetiva de analisar a qualidade dos resultados providos pelo método proposto. Uma vez que não existe uma verdade absoluta e conhecida sobre quem são de fato os usuários influentes e os conteúdos relevantes disseminados através da rede, nós utilizamos a tarefa de recomendação como uma forma de avaliação da nossa técnica. Nossa premissa é que a eficácia da nossa técnica em recomendar usuários e conteúdos efetivamente para outros usuários é uma evidência da sua eficácia no descobrimento de usuários influentes e de conteúdos relevantes. Finalmente, promovemos ainda um estudo de caso, onde aplicamos o método proposto em uma aplicação Web real.

\section{Trabalhos Relacionados}

Nesta seção, revisamos brevemente as áreas que tratam de influência, recomendação e abordagens relacionadas ao algoritmo Pagerank, que são fundamentos do nosso trabalho.

Influência A noção de influência é um conceito muito importante nas áreas de sociologia [15], comunicação e marketing. Em especial, com o surgimento e popularização das redes sociais, influência nesse contexto tem sido um tópico de muita atenção e pesquisa. Identificar quem são os usuários influentes, e como a informação relevante se propaga nas redes sociais são tarefas que, se bem resolvidas, trazem conhecimento estratégico para empresas de marketing, campanhas políticas e estudos sociológicos. Alguns exemplos são: identificar o melhor "ponto de partida" para campanhas de marketing no Facebook, ou caracterizar o surgimento de "fenômenos nacionais" em redes sociais, como a crítica ao comentarista Galvão Bueno espalhada no twitter, com a hashtag \#foragalvao. No âmbito político, é im- 
Uma Estratégia Baseada em Difusão de Informação para Determinação de Conteúdos Relevantes e Usuários Influentes em Redes Sociais.

portante discernir quem são os formadores de opinião, de forma a direcionar campanhas a esses indivíduos. Apesar da importância da noção de influência, não há um consenso na literatura sobre qual é a melhor forma de medir a influência de um determinado usuário. Várias métricas foram propostas: número de seguidores ou amigos, número de retweets ou citações, ou uma combinação destes [6, 19]. Aparentemente, a maioria dos trabalhos conclui que popularidade (i.e. número de seguidores no twitter, número de amigos no Facebook) não necessariamente implica em influência [6]. Um exemplo é o trabalho de [24], onde os autores utilizam a noção de passividade para ajudar na determinação da influência de um usuário. A maioria dos usuários do Twitter atua como consumidor passivo, ou seja, não disseminam nenhum conteúdo para a rede. Outros usuários, por sua vez, acrescentam muito conteúdo, mas poucos usuários reagem ao conteúdo publicado. Tais usuários não são considerados influentes. Os usuários considerados influentes são aqueles que conseguem "romper a passividade" de outros usuários, ou seja, que publicam conteúdo que é propagado por seus seguidores. Outras métricas de influência utilizam o algoritmo PageRank, discutido logo a frente. O trabalho de [13] propõe uma forma de predizer, quantitativamente, como e quando a informação se espalha nas redes sociais. Ele assume que a difusão de informação ocorre baseada em um modelo viral, mas tal ação depende da vontade individual humana. Experimentos realizados com dados provenientes de campanhas de marketing viral mostraram que o comportamento das pessoas é altamente heterogêneo, mesmo diante das mesmas decisões a serem tomadas [20]. Portanto, modelos que predizem o comportamento de usuários levando em consideração a média da população falham em explicar a dinâmica das redes sociais. Em [25], nós estudamos o problema de predizer relações de influência a partir de dados difundidos na rede. Tal trabalho serviu como maior motivação para o este estudo. Este trabalho estende uma versão preliminar deste trabalho [28], em particular adicionando um estudo de caso real, além de outras melhorias.

Recomendação Com o grande crescimento do volume de informação disponível ao usuário, tornou-se difícil a tarefa de encontrar conteúdo relevante e novo. Nesse contexto surgiram os sistemas de recomendação [23]. Dentre eles, se destacam os algoritmos baseados em filtragem colaborativa [18], que geram recomendações utilizando padrões de uso (exemplo: compras, avaliações), sem a necessidade de informação sobre o domínio ou os itens recomendados. No contexto específico do Twitter, vários trabalhos exploram recomendação, tanto de usuários quanto de tweets. O trabalho de [27], por exemplo, propõe um método personalizado por usuário, onde as recomendações feitas para um determinado usuário são escolhidas de forma a maximizar a probabilidade de que o usuário propague a informação recomendada. Outro exemplo é o trabalho de [12], em que o os autores propõem técnicas baseadas em conteúdo e em filtros colaborativos a fim de recomendar usuários a serem seguidos.

PageRank O algoritmo PageRank [5] assinala um valor de importância a páginas da Web, de forma que uma página $p$ tem um peso proporcional ao número e importância das páginas 
que apontam (através de hyperlinks) para $p$. Outro algoritmo que leva em conta a relação entre as páginas é o HITS (Hypertext Induced Topic Search), proposto em [17], que utiliza o conceito de autoridades e hubs. Intuitivamente, hubs são páginas que não são autoridades por si só, mas direcionam os usuários a páginas importantes. Páginas importantes (autoridades), por sua vez, são páginas que são apontadas por vários hubs diferentes. Em [8], Franceschet revisita os conceitos por trás do PageRank, ressaltando que a Web foi revolucionada pela idéia de introduzir a noção de um "índice de importância", que calibra o status de uma página utilizando apenas a topologia do grafo da Web. O trabalho revisita os conceitos sobre os quais o PageRank está fundamentado. O autor destaca, por exemplo, a sociometria como um antecessor notavelmente antigo, uma vez que sociologistas foram os primeiros a utilizar abordagens de rede para identificar propriedades de grupos relacionados. O conceito por trás era a mesma premissa do Pagerank: Uma pessoa tem prestígio se ela é endossada por pessoas prestigiosas. Com a popularização e sucesso dos conceitos utilizados pelo PageRank, vários trabalhos passaram a explorar a circularidade da rede a fim de calcular a importância dos seus nós. Baluja et al. utilizam Random Walks a fim de prover um método simples para propagar informações de preferência através de uma variedade de grafos, utilizando um estudo de caso que é a recomendação de vídeos para usuários no Youtube [4]. Tong et al. propõem um método dinâmico em [26] para monitorar a proximidade entre autores e conferências, também baseado em Random Walks. Por fim, os autores de [29] propõem o TwitterRank, que utiliza o PageRank para quantificar a influência de usuários no Twitter. Os autores utilizaram tanto a similaridade do conteúdo postado pelos usuários quanto a rede de seguidores, e conseguiram caracterizar a presença de homofilia no Twitter, além de propor uma nova forma de medir a influência de um usuário. Entretanto, o trabalho leva em conta apenas o tópico do conteúdo postado, desconsiderando os retweets, assim como os links formados entre usuários e conteúdo, como fazemos neste presente trabalho.

\section{Detecção de Conteúdo Relevante e Usuários Influentes}

Esta seção provê definições para os principais conceitos empregados neste trabalho.

\subsection{Relevância e influência}

Esta seção apresenta alguns conceitos importantes relacionados à identificação de conteúdos relevantes e usuários influentes com base em dados de difusão de informação. A idéia é associar os usuários influentes e conteúdo relevante através de uma definição circular, seguindo o seguinte princípio:

Um conteúdo é considerado relevante se ele é criado e propagado por usuários influentes, e usuários influentes criam conteúdo relevante. 
Uma Estratégia Baseada em Difusão de Informação para Determinação de Conteúdos Relevantes e Usuários Influentes em Redes Sociais.

Além disso, podemos reformular esse princípio de relevância global a fim de suportar funções de relevância e influência personalizadas da seguinte forma:

Um conteúdo c é considerado relevante para um dado usuário u se ele é criado e propagado por usuários que são influentes para u, e um usuário v é considerado influente para u se esse usuário $v$ cria conteúdo que é relevante para $u$.

Seja $C$ o conjunto de conteúdos e $U$ o conjunto de usuários. Nós definimos a relevância global de um conteúdo $c \in C$ como uma função $r(c)$. Além disso, definimos a influência global de um usuário $u \in U$ como uma função $p(u)$. Como se trata de uma métrica orientada ao comportamento do usuário, a importância global de $r(c)$ depende da relevância personalizada $r(c, u)$, que dá a relevância do conteúdo $c$ para o usuário específico $u$. No entanto, $r(c)$ também é afetada pela influencia dos usuários, ou seja quanto mais influentes forem os usuários para os quais $c$ é relevante, mais relevante será $c$. Portanto, a relevância do conteúdo é baseada na influência dos usuários. Da mesma forma, definimos a influência $p(u)$ de um usuário $u$ com base na relevância do conteúdo que ele produz. A função de influência personalizada $p\left(u_{i}, u_{j}\right)$ dá a influência de um usuário $u_{i}$ para um usuário $u_{j}$. Essas definições circulares são formalizadas na Seção 3.3

É interessante entender o problema de identificar os usuários influentes e conteúdo relevante sob uma perspectiva de recomendação. Um conteúdo que é relevante para alguns usuários deveria ser recomendado para tais usuários. Portanto, podemos aplicar as funções $r(c, u)$ e $p\left(u_{i}, u_{j}\right)$ em um contexto de recomendação. Ao avaliar a eficácia dessas funções, podemos avaliar a qualidade das funções $r(c)$ e $p(u)$. Esta abordagem torna-se especialmente útil quando não há informação sobre a relevância do conteúdo e a influência dos usuários que é um cenário muito frequente. Por esse motivo utilizamos a tarefa de recomendação como uma forma de avaliar a eficiência da nossa nova métrica.

\subsection{Dados de difusão de informação}

Nós chamamos de dados de difusão de informação o conjunto de ocorrências de um item de informação. Cada ocorrência de um item é definida como uma tupla na forma < $u, c, t>$, onde $u$ é um usuário do conjunto de usuários $U, c$ é um conteúdo do conjunto de conteúdos $C$, e $t$ é um instante do tempo. Portanto, os dados de difusão de informação descrevem a associação entre usuários e conteúdo ao longo do tempo.

A Figura 1(a) mostra um exemplo ilustrativo de um conjunto de dados de difusão de informação onde $U=\left\{u_{0}, u_{1}, u_{2}, u_{3}\right\}, C=\left\{c_{0}, c_{1}, c_{2}\right\}$ durante o intervalo de tempo $\left[t_{0}, t_{5}\right]$. Considerando-se o Twitter como exemplo, $U$ representa o conjunto de usuários, $C$ representa o conteúdo postado pelos usuários (tweets, URLs, hashtags), e os instantes de tempo são definidos de acordo com o momento das postagens. Da mesma forma, $U$ pode ser um conjunto de blogueiros que postam conteúdo (URLs, palavras-chave, temas) ao longo 
Uma Estratégia Baseada em Difusão de Informação para Determinação de Conteúdos Relevantes e Usuários Influentes em Redes Sociais.

\begin{tabular}{|l|}
$u_{0}, c_{0}, t_{0}$ \\
$u_{0}, c_{1}, t_{1}$ \\
$u_{1}, c_{0}, t_{2}$ \\
$u_{1}, c_{2}, t_{3}$ \\
$u_{2}, c_{1}, t_{4}$ \\
$u_{3}, c_{2}, t_{5}$ \\
\hline
\end{tabular}

(a) Dados

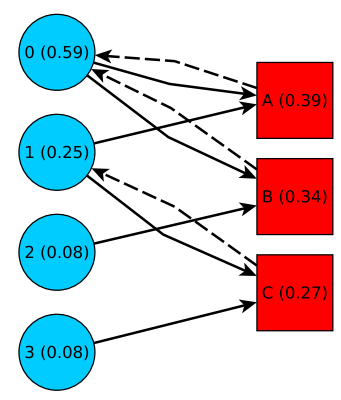

(b) Modelo de difusão

Figura 1. Modelagem de dados de difusão: Visão geral. Os dados em 1(a) representam usuários $u$ que postaram conteúdos $c$ nos tempos $t$. Esses dados são utilizados para gerar o grafo bipartido 1(b) Os usuários são representados por círculos e os conteúdos pelos quadrados. As setas contínuas ligam o usuário à cada conteúdo que ele criou ou propagou, e as setas tracejadas ligam o conteúdo de volta apenas ao usuário que o criou. Os números dentro de cada vértice indicam os valores de influência/relevância calculados pela nossa técnica.

do tempo. Dados de difusão de informação aparecem em muitos outros cenários da vida real, especialmente em aplicações de mídia social. É importante notar que a nossa definição de dados de difusão de informação não leva os relacionamentos socias entre os usuários da rede em consideração. Ou seja, não temos qualquer informação sobre amizades, seguidores ou qualquer outro tipo de relação que pode ser considerado como um meio para a difusão da informação. Dada a dificuldade de conseguir esses dados em larga escala, obter essas informações pode ser uma limitação para a aplicação de trabalhos que utilizem a rede social em situações reais, situação que não ocorre no presente trabalho.

\subsection{Modelo de Difusão de Informação}

Nosso modelo é baseado em um grafo bipartido $G(U, C, F, E)$ que associa os usuários à conteúdos através de dois conjuntos de arestas, $F$ e $E$. Para cada usuário $u \in U$ e conteúdo $c \in C$, existe uma aresta direcionada $(u, c) \in F$ se o usuário $u$ postou o conteúdo $c$, ou seja, se ele é o criador (primeira pessoa a postar aquele conteúdo) ou se ele propaga esse mesmo conteúdo posteriormente na rede (no caso do Twitter, isso equivaleria a um retweet). As arestas em $F$ atribui relevância ao conteúdo com base na influência do usuário. Além disso, existe uma aresta direcionada $(c, u) \in E$ que parte de um conteúdo para o usuário que o criou, e cada conteúdo tem apenas um criador. Arestas em $E$ dão crédito aos usuários de acordo com 
Uma Estratégia Baseada em Difusão de Informação para Determinação de Conteúdos Relevantes e Usuários Influentes em Redes Sociais.

a relevância do conteúdo que eles criam. A Figura 1(b) apresenta o grafo bipartido construído a partir dos dados mostrados na Figura 1(a) Definimos a relevância do conteúdo $r(c)$ como a frequência relativa em que um random surfer [5] que começa a partir de um nó de usuário arbitrário e, navegando através do gráfico bipartido $G(U, C, F, E)$, atinge um determinado conteúdo $c$. Já a relevância personalizada $r(c, u)$ começa a partir de um determinado usuário $u$ em vez de um usuário arbitrário. De um modo semelhante, a influência de um usuário $p(u)$ é a frequência relativa que o random surfer visita um determinado usuário e pode ser personalizado, iniciando a partir de um usuário particular. Para dar uma visão mais realista sobre o nosso modelo, vamos considerar o Twitter como um cenário de exemplo. $\mathrm{O}$ algoritmo seguido pela nosso random surfer, com base em dados do Twitter, pode ser descrito como se segue:

1. Seleciona um perfil de usuário arbitrário;

2. Escolhe um tweet aleatório ou retweet do usuário atual;

3. Seleciona o perfil do autor do tweet dado; e

4. Volta para o passo 2.

O gráfico bipartido $G(U, C, F, E)$ pode ser representado por duas matrizes, $M$ e $L$. A matriz $M=\left(m_{i, j}\right)$ é $|U| \times|C|$ e $m_{i, j}=1 / q_{i}$, onde $q_{i}$ é a quantidade de conteúdo que $u_{i}$ criou ou propagou. Além disso, $L=\left(l_{i, j}\right)$ é $|C| \times|U|$ e $l_{i, j}=1$ se o usuário $u_{j}$ criou o conteúdo $c_{i}$ ou $l_{i, j}=0$, caso contrário. Com base em $M$ e $L$, a função de relevância do conteúdo $r(c)$ e a função de influência do usuário $p(u)$ são definidas assim:

$$
\begin{aligned}
& r=p M \\
& p=r L
\end{aligned}
$$

onde $r$ é um vetor de relevância do conteúdo (ou seja, $r_{i}$ é a relevância do conteúdo $c_{i}$ ) e $p$ é um vetor de relevância do usuário (ou seja, $p_{j}$ é a influência do usuário $u_{j}$ ). Nessa definição, assumimos que já temos um dos vetores $(r$ ou $p$ ), a fim de calcular um a partir do outro, o que não acontece na realidade. Contudo, $r$ e $p$ podem ser calculados recursivamente:

$$
\begin{aligned}
& r^{(k)}=r^{(k-1)} L M \\
& p^{(k)}=p^{(k-1)} M L
\end{aligned}
$$

onde $k \geq 0$ e $r^{(0)}$ e $p^{(0)}$ são vetores uniformes $\mathrm{S}^{3}$ Esse modelo apresenta dois problemas importantes: (1) A possível presença de usuários dangling e (2) a possível existência de

\footnotetext{
${ }^{3}$ Em um vetor uniforme, todos os valores são iguais e a soma deles é 1
} 
buckets. Um usuário dangling é um usuário que nunca propaga conteúdo de outros usuários. Considerando a metáfora do random surfer, o surfer ficará preso sempre que um usuário dangling $u$ é atingido pois ele sempre seguirá arestas para o conteúdo gerado por $u$ e depois consequentemente voltará para $u$. O PageRank também precisa lidar com páginas dangling e nós aplicamos uma solução semelhante aqui. Criamos uma aresta $(u, c)$ de cada usuário dangling para um conteúdo "fantasma" $c$ e adicionamos uma aresta $(c, u)$ a partir do conteúdo fantasma para cada usuário $u \in U$. Como consequência, garantimos que o random surfer conseguirá, a partir de um usuário dangling, chegar a qualquer outro usuário em $U$. No gráfico mostrado na Figura 1(b), $u_{0}$ é um usuário dangling. Um bucket é um subgrafo fortemente conexo do grafo bipartido. Quando o random surfer atinge um bucket, ele não é capaz de deixá-lo. Podemos ver um usuário dangling como se fosse um bucket de tamanho 1. A fim de evitar que o random surfer fique preso em buckets, podemos adicionar um mecanismo de amortecimento ao nosso modelo. Esse mecanismo determina uma pequena probabilidade $d$ do random surfer pular do usuário atual para um conteúdo aleatório ou vice-versa. Nós adicionamos esse mecanismo na definição de $r$ e $p$ da seguinte forma:

$$
\begin{aligned}
& r^{(k)}=c r^{(k-1)} L M+(1-c) u \\
& p^{(k)}=c p^{(k-1)} M L+(1-c) u
\end{aligned}
$$

em que $u$ é um vetor uniforme. Podemos reformular as equações acima algebricamente a fim de obter as suas soluções exatas de uma forma não recursiva:

$$
\begin{aligned}
& r=(1-d) u(I-d L M)^{-1} \\
& p=(1-d) u(I-d M L)^{-1}
\end{aligned}
$$

Na próxima seção, vamos discutir por que essa formulação algébrica não é computacionalmente eficiente. Duas questões mais importantes neste momento são: (1) Será que essas equações têm uma solução? e (2) Essas soluções são únicas? A resposta afirmativa para a primeira pergunta vem do fato de que as matrizes $M L$ e $L M$ são estocásticas. De fato, sabe-se que o produto de duas matrizes estocásticas é sempre uma matriz estocástica. Além disso, uma combinação linear de duas matrizes estocásticas é também estocástica. Em relação à pergunta 2 , podemos mostrar que as nossas equações têm uma solução única, baseada no teorema de Perron-Frobenius [10, 8]. O teorema de Perron-Frobenius diz que se uma matriz $A$ é irredutível (ou seja, se seu gráfico associado é fortemente conectado) e também quadrada não negativa, então a equação $x A=r x$, onde $x>0$ e $\sum_{i} x_{i}=1$, tem uma única solução. Como $M, L$, e $u$ são não-negativos, nossas equações tem matrizes não negativas. Além disso, a remoção de usuários dangling e de buckets garante que $M L$ e $L M$ são irredutíveis. Na Figura 1(b), calculamos os valores da influência do usuário e da relevância do conteúdo usando $d$ igual a 0,85 . Podemos notar que o usuário mais influente é $u_{0}\left(p\left(u_{0}\right)=0.59\right)$, pois os dois conteúdos produzidos por $u_{0}\left(c_{0}\right.$ e $\left.c_{1}\right)$ são propagados por dois usuários $\left(u_{1}\right.$ e $\left.u_{2}\right)$. Os 
Uma Estratégia Baseada em Difusão de Informação para Determinação de Conteúdos Relevantes e Usuários Influentes em Redes Sociais.

conteúdos produzidos por $u_{1}$ também são propagados por dois usuários, mas esses usuários são menos influentes do que os usuários que propagam o conteúdo a partir de $u_{0}$. Portanto, $u_{1}$ é menos influente que $u_{0}$. O conteúdo mais relevante é $c_{0}$ porque ele foi difundido por dois usuários influentes $\left(u_{0} \mathrm{e} u_{1}\right)$. Embora $c_{2}$ também seja difundido por dois usuários, esses usuários não são tão influentes como os associados a $c_{0}$. A elaboração de valores personalizados de relevância de conteúdo $(r(c, u))$ e influência de usuário $\left(p\left(u_{i}, u_{j}\right)\right)$ em nosso modelo é simples. Nestes cenários, em vez de iniciar a partir de um usuário arbitrário, vamos supor que o random surfer começa a partir de um usuário específico para qual o modelo está sendo personalizado. Da mesma forma, em vez de saltar para um conteúdo aleatório com uma probabilidade não-zero, o random surfer sempre salta de volta para esse nó específico. Esse comportamento pode ser induzido substituindo o vetor uniforme $u$ por um vetor $1_{i}$, que é um vetor com todos os elementos iguais a 0 , com exceção da posição $i$ igual a 1 , onde $u_{i}$ é o usuário para o qual o modelo está sendo personalizado.

\subsection{Solução Eficiente}

$\mathrm{Na}$ seção anterior, descrevemos as equações que definem influência do usuário e relevância do conteúdo no nosso modelo. Para aplicar esse modelo em cenários reais de mídia social, com grande volume de usuários e conteúdo, precisamos resolver tais equações de forma eficiente. Em situações reais, as matrizes $M$ e $L$ tendem a ser muito grande e esparsas. Portanto, uma solução eficiente para nosso modelo deve levar em consideração essas propriedades. Como mostrado nas Equações 1 e 2, podemos calcular os vetores $r$ e $p$ invertendo uma matriz $|U| \times|U|$ e uma matriz $|C| \times|C|$. Como a inversão de uma matriz $n \times n$ tem custo $O\left(n^{3}\right)$, calcular os valores exatos de $r$ e $p$ não é viável em situações reais. No entanto, o método da potências [22, 8], que é um método de iteração rápido para calcular o autovalor e autovetor dominante de uma matriz, pode ser aplicado no cálculo de $r$ e $p$. O Algoritmo 1 descreve o método da potências. Ele recebe duas matrizes $\left(Z_{1}\right.$ e $\left.Z_{2}\right)$ e repetidamente itera sobre a solução $g$, que é iniciada como uniforme, até que um determinado número de iterações $k$ seja atingido. Se $Z_{1}=M$ e $Z_{2}=L$, o método nos dá o vetor de influência $(p)$. Por outro lado, se $Z_{1}=L$ e $Z_{2}=M$, ele nos dá o vetor de relevância $(r)$. Conforme descrito na seção anterior, podemos calcular os valores personalizados de influência e relevância para um usuário $u_{i}$, substituindo o vetor uniforme $U$ por um vetor $1_{i}$ que tem 1 na $i$-ésima posição e 0 nas posições restantes. Além de aplicar o método de potências para calcular a influência e relevância, fazemos uso de representações esparsas das matrizes $M$ e $L$, com o objetivo de reduzir a quantidade de memória e o tempo de execução para computar $r$ e $p$. Mais especificamente, representamos matrizes no formato de coordenadas. Valores são armazenados numa lista de tuplas (linha, coluna, valor), onde apenas tuplas com valores diferentes de zero 
Uma Estratégia Baseada em Difusão de Informação para Determinação de Conteúdos Relevantes e Usuários Influentes em Redes Sociais.

são inseridas.

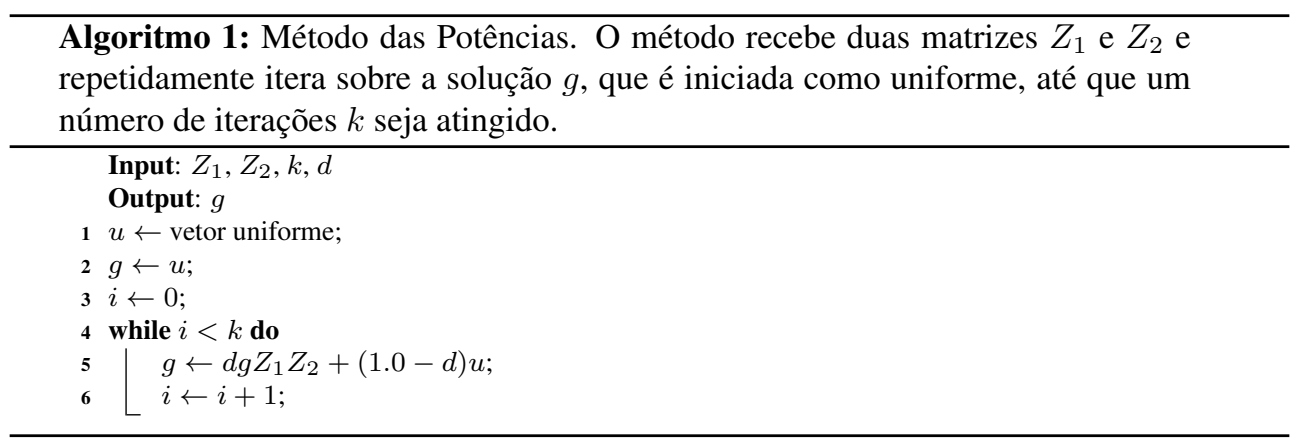

\section{Resultados Experimentais}

Nesta seção, iremos apresentar os principais resultados experimentais deste trabalho. Nosso objetivo é avaliar o modelo para a análise de influência de usuários e relevância de conteúdo utilizando dados reais.

\subsection{Bases de dados}

Nós utilizamos duas bases de dados obtidas a partir do Twitter ${ }^{4}$ A coleta de dados foi realizada através da API de coleta em modo streaming do Twitter ${ }^{5}$ Para cada base de dados, conjuntos de palavras-chave relevantes em um dado contexto foram selecionadas manualmente. Os contextos utilizados neste trabalho são automóveis e as eleições americanas para presidente. A Tabela 1 sumariza as principais propriedades das bases de dados utilizadas. De maneira geral, nós consideramos tweets como conteúdo e retweets como forma de propagação de conteúdo através de usuários.

\begin{tabular}{l|crrc}
\hline nome & \#usuários & \#tweets & \#RTs & período de coleta \\
\hline Eleições & 529.630 & 369.287 & 1.368 .080 & $31 / 12 / 2011-31 / 01 / 2012$ \\
Carros & 127.106 & 53.670 & 138.352 & $10 / 01 / 2012-05 / 03 / 2012$ \\
\hline
\end{tabular}

Tabela 1. Bases de dados

A Figura 2 mostra uma breve caracterização de importantes aspectos da base de dados Eleições. Mais especificamente, são apresentadas as distribuições do número de tweets por

4 http://twitter.com/

5 https://dev.twitter.com/docs/streaming-api 
Uma Estratégia Baseada em Difusão de Informação para Determinação de Conteúdos Relevantes e Usuários Influentes em Redes Sociais.

usuário, de retweets (RTs) por usuário e de retweets por tweet. Podemos notar que, como era esperado, a maior parte das distribuições aparenta seguir uma lei de potências, ou seja, grande concentração de popularidade e atividade de usuários. Realizamos a mesma caracterização para a base de dados de Carros, a qual apresentou propriedades muito semelhantes.

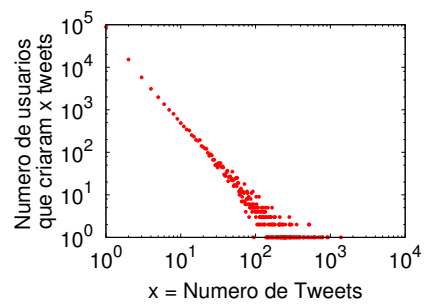

(a) Distribuição de tweets por usuário

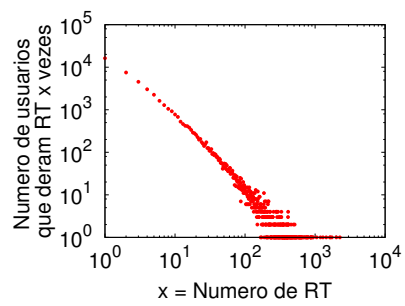

(b) Distribuição de RTs por usuário

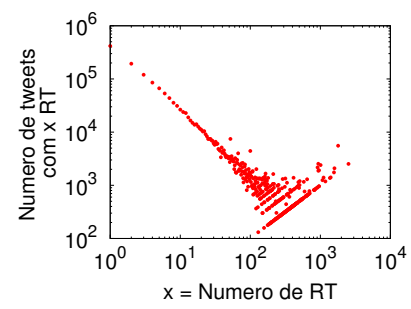

(c) Distribuição de RTs por tweet

Figura 2. Caracterização da Base de dados Eleições

\subsection{Recomendação de conteúdo}

Apesar de se tratar de um problema bastante estudado na literatura, não existem maneiras efetivas de avaliar a qualidade de um método que identifica usuários influentes. Além disso, como nossa definição de relevância esta fortemente associada à de influência, analisar a eficácia do modelo proposto se torna um desafio. Neste trabalho, nós propomos uma interpretação do conceito de relevância com base no problema de recomendação. Dessa forma, um conteúdo é considerado relevante em geral, se ele é relevante para muitos usuários, especialmente os mais influentes. Isso permite que a avaliação do modelo proposto no contexto de recomendação de conteúdo gere evidências da sua qualidade na identificação de conteúdo relevante e usuários influentes. Em outras palavras, se a versão personalizada do modelo é capaz de recomendar conteúdo para usuários de forma acurada, então podemos inferir que ele também é capaz de identificar conteúdo relevante em geral e, por consequência 

Relevantes e Usuários Influentes em Redes Sociais.

das premissas do modelo, avaliar a influência de usuários.

Os experimentos para esta avaliação foram realizados da seguinte forma: Para cada tweet da base de dados, seus retweets foram divididos entre dados de treino e teste numa razão $50 \% / 50 \%$, considerando a ordem de ocorrência. As primeiras ocorrências de cada tweet foram inserídas na base de treino e o restante na base de teste. Os dados de treino foram utilizados na construção de modelos de recomendação a serem avaliados utilizando os dados de teste. Nós consideramos como baseline uma técnica tradicional de recomendação de conteúdo denominada Filtragem Colaborativa. A idéia básica desta técnica é se basear na semelhança entre interesses de usuários para recomendar novos itens. Dois usuários $u_{1} \mathrm{e}$ $u_{2}$ são considerados semelhantes se eles compartilham interesses em comum e o modelo de recomendação identifica potenciais itens consumidos por $u_{1}$ a serem recomendados a $u_{2} \mathrm{e}$ vice-versa. Existem diversas variações de filtragem colaborativa. Neste trabalho, nós empregamos uma estratégia denominada recomendação de itens baseada em vizinhos mais próximos com pesos (do inglês weighted item $K N N$ ). A implementação empregada foi obtida a partir da biblioteca de recomendação MyMediaLit 6 . Todos os parâmetros foram utilizados em sua configuração default. A métrica de avaliação utilizada neste trabalho é a curva ROC (do inglês Receiver Operating Characteristic). Uma curva ROC mostra como a taxa de verdadeiros positivos (TPR) e falsos positivos (FPR) varia ao longo do intervalo de scores gerado por um dado modelo. Em geral, é esperado que modelos sejam mais efetivos no topo das previsões, ou seja, no início da curva ROC. A medida da qualidade de um modelo é obtida através do cálculo da área da curva ROC, denominada AUC (do inglês Area Under the Curve).

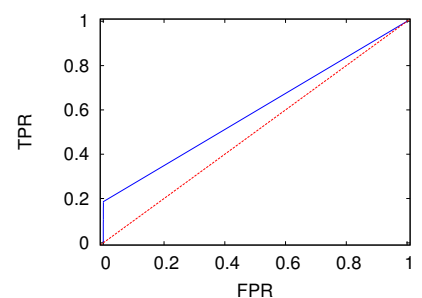

(a) WIKNN, $\mathrm{AUC}=0.59$

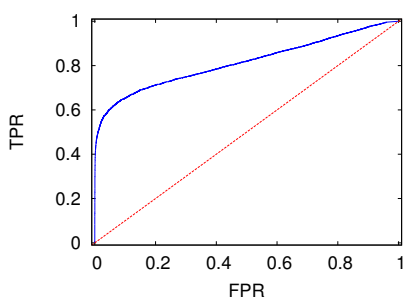

(b) $\mathrm{MDC}, \mathrm{AUC}=0.81$

Figura 3. Curva ROC para uma técnica de filtragem colaborativa (WIKNN) e para nosso modelo baseado em difusão de conteúdo (MDC)

Nesta avaliação foi considerada apenas a base de dados Carros. A Figura 3 mostra uma comparação entre o modelo baseado em difusão de conteúdo e uma técnica de filtragem colaborativa. Podemos notar que nosso modelo apresenta uma acurácia $37 \%$ superior ao

6ttp://www.ismll.uni-hildesheim.de/mymedialite/index.html 
Uma Estratégia Baseada em Difusão de Informação para Determinação de Conteúdos Relevantes e Usuários Influentes em Redes Sociais.

\begin{tabular}{|l|c|c|}
\hline tweet & relevância & usuário \\
\hline $\begin{array}{l}\text { Dia sem Globo' faz emissora registrar o dobro da Record e o triplo do SBT em } \\
\text { audiencia - Parabens aos envolvidos. }\end{array}$ & 0.003 & kibeloco \\
\hline $\begin{array}{l}\text { Quanto a Fiat pagou para mostrar que esse carro de bosta pequeno e que o porta- } \\
\text { malas é difícil de abrir? \#BBB12 }\end{array}$ & 0.002 & kibeloco \\
\hline $\begin{array}{l}\text { Delegado passa 4 horas ouvindo BBBs - Quatro horas? Coitado! Fez prova de } \\
\text { resisteancia e nao levou nem um Fiat... }\end{array}$ & 0.002 & kibeloco \\
\hline $\begin{array}{l}\text { "Tem uns professores que acham que os alunos tem problemas de audicao, so } \\
\text { pode. }\end{array}$ & 0.002 & PiadasDeAluno \\
\hline $\begin{array}{l}\text { FALA: GATA VOCE NAO é DONA DA FIAT, MAIS VOCE FAZ MEU STILO } \\
\text { AS MINA PIRA E FICA SEM JEITO D + }\end{array}$ & 0.002 & AsMinaaPira \\
\hline
\end{tabular}

Tabela 2. Tweets mais relevantes da base de dados Carros

\begin{tabular}{|l|c|c|}
\hline tweet & relevância & usuário \\
\hline $\begin{array}{l}\text { Rosa Parks sat, so that Dr. Martin Luther King, Jr. could walk, so that Barack } \\
\text { Obama could run, so that the next generation, us, could fly. }\end{array}$ & 0.004 & mind \\
\hline Rick Santorum's stance on homosexuality is so fucking gay. & 0.003 & SethMacFarlane \\
\hline I feel like Newt Gingrich is what Justin Bieber will look like old. & 0.002 & SethMacFarlane \\
\hline $\begin{array}{l}\text { Rick Santorum seems so homophobic that I'm surprised he even allows another } \\
\text { man to vote for him. }\end{array}$ & 0.002 & GarryShandling \\
\hline It's illegal for prisoners to vote, but they can run for President of the United States! & 0.002 & WTFuckFacts \\
\hline
\end{tabular}

Tabela 3. Tweets mais relevantes da base de dados Eleições

baseline. A explicação para a superioridade do nosso modelo é a sua capacidade de generalizar relacionamentos entre usuários que não compartilham tweets em comum através de random walk. Dessa forma, nosso modelo é mais efetivo em lidar com a esparsidade dos dados do que técnicas tradicionais. No modelo, o número de iterações e o valor do damping factor $(d)$ foram definidos como 10 e 0.85 , respectivamente.

\subsection{Conteúdo relevante e usuários influentes}

Esta seção apresenta os tweets relevantes e usuários influentes identificados pelo nosso modelo a partir das bases de dados Carros e Eleições. O objetivo é mostrar que tal modelo obtém resultados que são semanticamente válidos dentro dos contextos considerados.

A Tabela 2 mostra os tweets mais relevantes identificados na base Carros. Os tweets são mostrados da forma que aparecem na base de dados, apenas alguns caracteres especiais foram removidos. Devido a uma falha na coleta, alguns dos tweets citados não são sobre automóveis. O tweet mais relevante, por exemplo, foi coletado devido a presença do termo 'audiência', que a API de coleta confunde com o termo 'audi'. Em geral, podemos notar que os tweets mais relevantes têm papel humorístico e foram criados por usuários relacionados a esse gênero, como é o caso do usuário kibeloco. Esses tweets alcançam um grande número de retweets, o que explica a identificação dos mesmos como relevantes. Resultados semelhantes foram encontrados na análise da base de dados Eleições, como mostrado na Tabela 3 . 
Uma Estratégia Baseada em Difusão de Informação para Determinação de Conteúdos Relevantes e Usuários Influentes em Redes Sociais.

\begin{tabular}{|c|c|}
\hline usuário & relevância \\
\hline Estadao & 0.008 \\
\hline VEJA & 0.008 \\
\hline viacertanatal & 0.007 \\
\hline kibeloco & 0.007 \\
\hline aguinaldaosilva & 0.006 \\
\hline rdloficial & 0.006 \\
\hline viacertaRN & 0.003 \\
\hline JovemPanBH & 0.003 \\
\hline kiamotorsbrasil & 0.003 \\
\hline
\end{tabular}

(a) Carros

\begin{tabular}{|c|c|}
\hline usuário & influência \\
\hline BorowitzReport & 0.020 \\
\hline LOLGOP & 0.012 \\
\hline BreakingNews & 0.008 \\
\hline RonPaul & 0.008 \\
\hline robdelaney & 0.007 \\
\hline thinkprogress & 0.007 \\
\hline SethMacFarlane & 0.006 \\
\hline AP & 0.06 \\
\hline rationalists & 0.005 \\
\hline
\end{tabular}

(b) Eleições

Figura 4. Usuários mais relevantes identificados

A Figura 4 mostra os usuários mais influentes na base Carros (Figura 4(a) e Eleições (Figura 4(b)]. De maneira geral, os usuários mais influentes são aqueles que obtiveram um maior número de retweets, especialmente retweets de outros usuários influentes, e compreendem perfis de agências de notícias (e.g., Estadao, AP), perfis humorísticos(e.g., kibeloco, LOLGOP), e personagens atuantes (e.g., aguinaldaosilva, RonPaul). É interessante notar que tais resultados são diferentes dos de técnicas simples como a contagem do número de retweets ou do número de seguidores dos usuários.

\section{Estudo de Caso: Observatório das Eleições}

Nesta seção, apresentamos um estudo de caso no qual aplicamos a técnica proposta em um cenário real: o Observatório das Eleiçõe 7 A Seção 5.1 apresenta o contexto em que nosso estudo de caso foi concebido e a motivação para o mesmo. Na Seção 5.2, descrevemos sua implementação e apresentamos nas Seções 5.3 e 5.4 uma descrição da base de dados e uma análise qualitativa do método, respectivamente. Por fim, a Seção 5.5 apresenta casos em que uma aplicação semelhante é plausível.

\subsection{Contexto e Motivação}

O Observatório das Eleições foi lançado em 2010, quando acompanhou os conteúdos relacionados à disputa presidencial brasileira, e pertence a um projeto mais amplo, denominado Observatório da Web. O Observatório da Web é uma ferramenta gratuita dedicada ao monitoramento de importantes fatos, eventos e entidades na rede mundial de computadores em tempo real. Com o objetivo principal de descobrir o que as pessoas publicam na internet acerca do processo eleitoral, a Universidade Federal de Minas Gerais criou o Observatório das Eleições de 2012 (OE2012), que monitorou as eleições municipais brasileiras

7 www.observatorio.inweb.org.br/eleicoes/destaques 
Uma Estratégia Baseada em Difusão de Informação para Determinação de Conteúdos Relevantes e Usuários Influentes em Redes Sociais.

deste mesmo ano. Desenvolvidos sob a coordenação do Departamento de Ciência da Computação da UFMG, os softwares responsáveis pelo funcionamento do site promovem a mineração dos dados provenientes da web relacionados a candidatos e a temas que estejam em pauta em portais de notícias e em redes sociais e canais diversos, em quatorze capitais espalhadas pelas cinco regiões do país.

O período de campanha eleitoral para as eleições municipais em 2012 teve início oficial no dia seis de julho. A partir desta data, os candidatos puderam ganhar as ruas em busca de apoiadores e votos, além de terem suas agendas de campanha divulgadas pelos noticiários. Mas o "período eleitoral" começa de fato, para grande parte do eleitorado, apenas quando o horário gratuito de propaganda eleitoral entra na programação das emissoras de rádio e de televisão abertas. A partir deste momento, é praticamente impossível não ouvir falar em eleições. A propaganda eleitoral gratuita em 2012 começou no dia 21 de agosto e foi, a partir dessa data, que se intensificou os esforços para influenciar a decisão do eleitor.

Diante de tantos esforços para influenciar eleitores, descobrir quem são os usuários influentes e o que eles estão dizendo a respeito dos candidatos e partidos mostra-se uma informação preciosa. Como apresentado na Seção 3.1. a técnica proposta neste artigo nos permite descobrir essas duas informações de forma computacionalmente eficiente. Dado o valor dessas informações, o OE2012 agregou às suas ferramentas de análise a métrica apresentada neste artigo, gerando como resultado um ranking diário de usuários considerados influentes no que diz respeito às eleições. Esse ranking permite visualizar os usuários mais influentes no dia, bem como seu histórico ao longo dos dias observados. Também é possível filtrar o ranking para uma cidade específica dentre as 14 escolhidas para o OE2012.

\subsection{Descrição da Implementação}

Nesta seção descrevemos como o OE2012 foi implementado. A Figura 5 mostra uma ilustração do fluxo que os dados percorrem desde a coleta até sua apresentação ao usuário. 


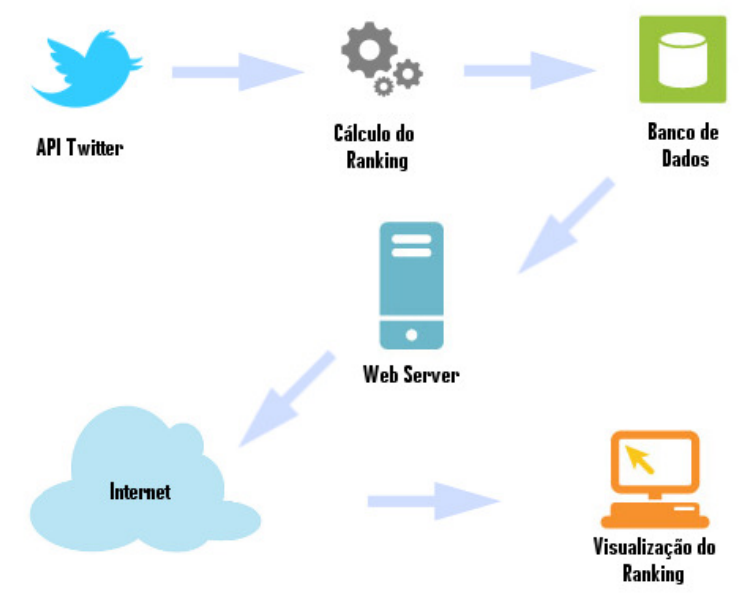

Figura 5. Fluxo dos Dados

A primeira etapa diz respeito à coleta dos dados. Semelhantemente como descrito na Seção 4.1. os dados para o monitoramento foram obtidos do Twitter, através de sua API. Nesta etapa, os dados são recebidos do Twitter, e palavras chaves que foram selecionadas manualmente são usadas para filtrar os dados relacionados às eleições. Elas também são utilizadas para segregar os dados em cada uma das 14 cidades acompanhadas pelo OE2012.

De posse dos dados relacionados às eleições e catalogados por cidades, o próximo passo é o cálculo da influência de cada usuário através da técnica apresentada. Uma vez pré-processados, estes dados são enviados ao nosso algoritmo, que cria o modelo de difusão de informação e calcula a influência de cada usuário. Com essas informações, os rankings são gerados, e armazenados então em um banco de dados. Diariamente, quinze rankings são processados. São quatorze rankings para as cidades acompanhadas e um que une todo o país. Após armazenados no banco de dados, essas informações ficam acessíveis através do servidor Web, responsável pela interface de iteração com o usuário. 
Uma Estratégia Baseada em Difusão de Informação para Determinação de Conteúdos Relevantes e Usuários Influentes em Redes Sociais.

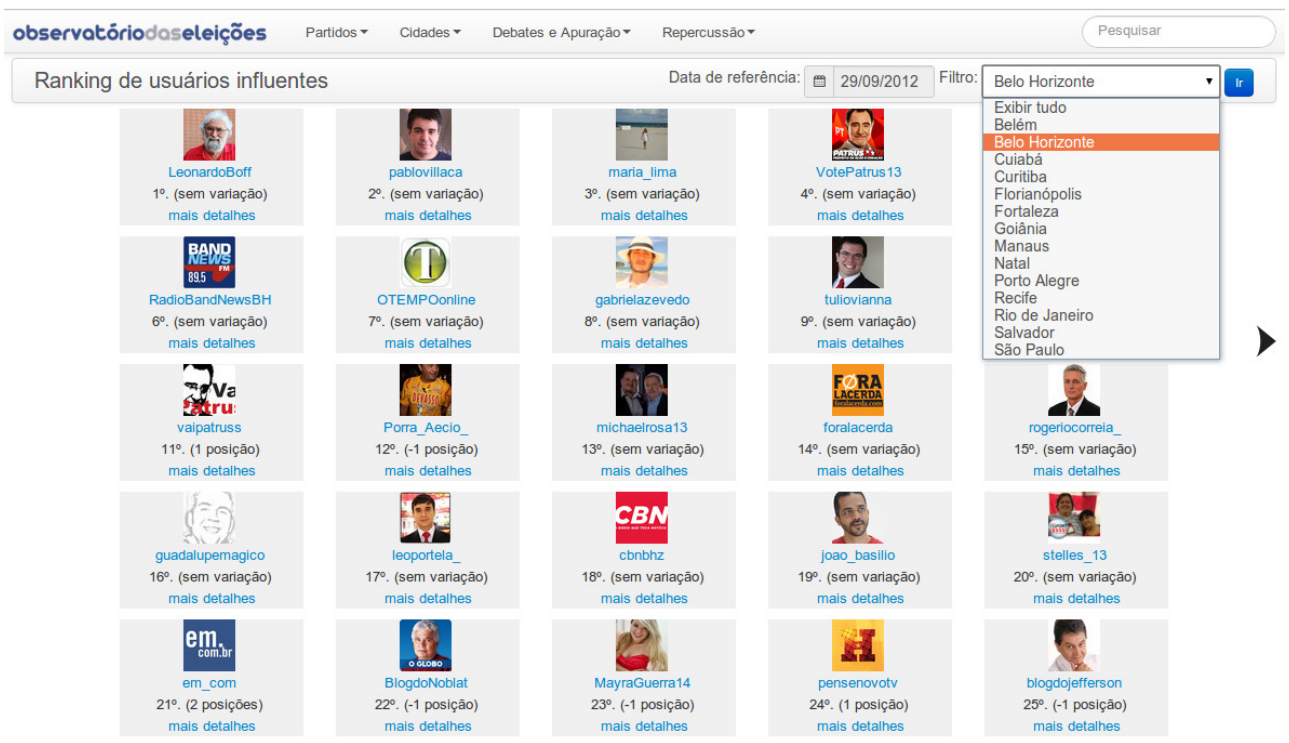

Figura 6. Interface web que apresenta o ranking de usuários de acordo com os filtros selecionados

A interface, apresentada na Figura 6, proporciona aos usuários formas simples de aplicar os filtros desejados: seja por data ou por cidade. Além dessas duas opções, existe ainda a possibilidade de verificar o histórico temporal de cada perfil do ranking, através da opção "mais detalhes". Essa análise, conforme mostrado na Figura 7 apresenta a evolução temporal da posição ocupada pelo perfil sendo analisado no ranking de influência. Dessa forma, usuários podem acompanhar como a influência de um perfil variou ao longo do período eleitoral.

\subsection{Descrição da Base de Dados}

A Tabela 4 apresenta um resumo da base de dados coletada através da metodologia descrita na Seção 5.2 Como pode ser visto, a base conta com quase cem mil usuários distintos, e quase meio milhão de tweets criados e/ou propagados. Após um pré-processamento, obtemos 62.296 usuários, sendo que, dentre esses, apenas 60 deles ocuparam alguma vez as 10 primeiras posições. Esses 60 usuários, que são menos de $0.01 \%$ de todos os usuários, foram responsáveis por 6.487 de todos os conteúdos da base, o que representa 1,35\% de todo o conteúdo criado/propagado.

A Figura 8 mostra uma breve caracterização dos dados apresentados ao usuário. Como 
Uma Estratégia Baseada em Difusão de Informação para Determinação de Conteúdos Relevantes e Usuários Influentes em Redes Sociais.

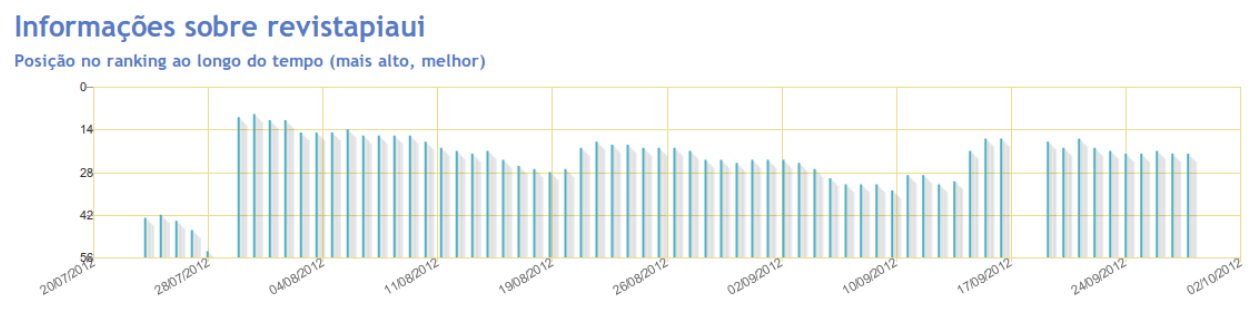

Figura 7. Interface que apresenta um histórico da evolução temporal das posições ocupadas por um dado perfil de usuário no ranking de influência. Nesse exemplo, pode-se perceber que o perfil revistapiaui apareceu muito entre as 30 primeiras posições.

\begin{tabular}{l|cccc}
\hline nome & \#usuários & \#tweets & \#RTs & período de coleta \\
\hline Eleições 2012 & 97.469 & 125.301 & 354.829 & $6 / 07 / 2012-29 / 09 / 2012$ \\
\hline
\end{tabular}

Tabela 4. Base de dados

descrito anteriormente, o usuário têm à sua disposição 14 capitais que foram monitoradas durante o período de eleições, e ainda um ranking global, sendo que todos medem a influência de cada usuário no contexto explorado. A influência é calculada de acordo com a nova métrica apresentada neste trabalho. Em cada gráfico desta figura, para cada posição do ranking, temos o número de usuários que sempre ocupou uma posição mais ao topo ou igual a ela, durante todo o período monitorado.

A Figura 8(a) apresenta os resultados para o ranking geral. Podemos observar que a curva gerada apresenta uma distribuição que segue a Lei de Potências. Essa distribuição é comum em análises de redes sociais, e neste caso mostra que muito poucos usuários estão sempre nas posições do topo, e a maioria dos usuários ocupa posições mais inferiores do ranking. É interessante notar ainda que quase todas as cidades possuem um comportamento semelhante. 
Uma Estratégia Baseada em Difusão de Informação para Determinação de Conteúdos Relevantes e Usuários Influentes em Redes Sociais.

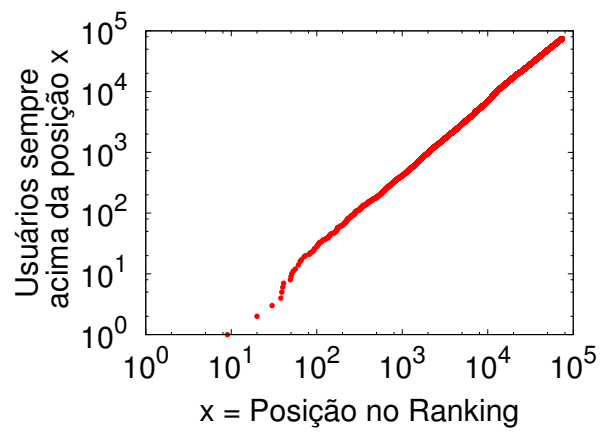

(a) Geral

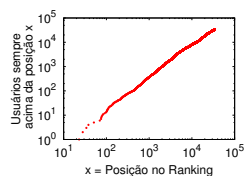

(b) São Paulo

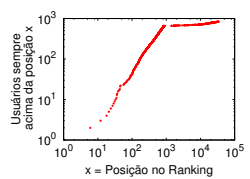

(f) Belém

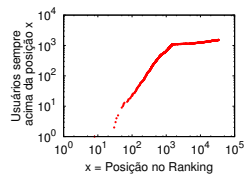

(j) Goiânia

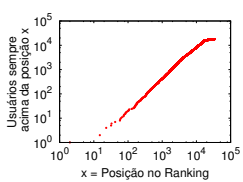

(c) Rio de Janeiro

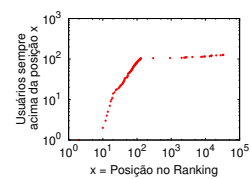

(g) Cuiabá

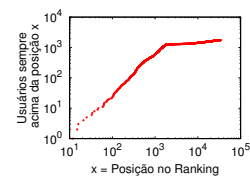

(k) Manaus

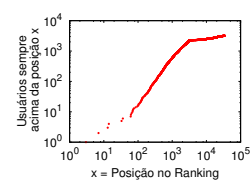

(n) Recife

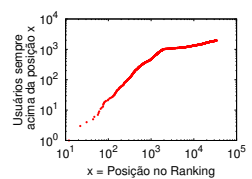

(d) Belo Horizonte

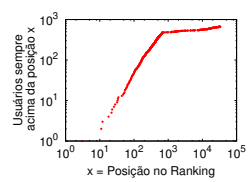

(h) Florianópolis

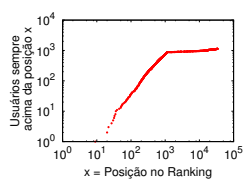

(1) Natal

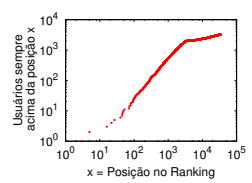

(o) Salvador

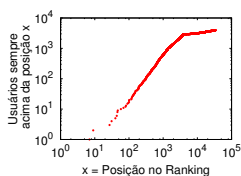

(e) Curitiba

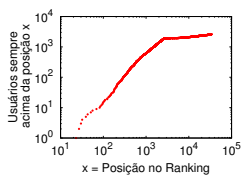

(i) Fortaleza

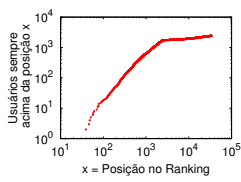

(m) Porto Alegre

Figura 8. Distribuição acumulada da posição mais baixa do ranking ocupada pelos usuários. Para cada posição do ranking, é mostrada o número de usuários que sempre apareceu pelo menos naquela posição (na posição X ou em uma acima dela) em qualquer um dos dias durante o período observado. Os gráficos mostram as 14 capitais monitoradas, além do ranking geral. Todos os gráficos estão em escala log-log 


\subsection{Análise}

Como já discutido anteriormente, não existe uma maneira definida para avaliar a qualidade dos resultados da técnica, uma vez que não existe um consenso na literatura de como medir a influência de um determinado usuário. Nos experimentos da Seção 4.2, optamos por tratar a avaliação como um problema de recomendação, validando quantitativamente a técnica proposta. Aqui, faremos uma análise qualitativa dos resultados baseada em intuição e conhecimentos gerais sobre o cenário político brasileiro.

É importante lembrar que o OE2012 oferece um ranking individual para cada uma das 14 cidades analisadas, e ainda um ranking global em que todas as cidades são avaliadas conjuntamente. Nos rankings individuais das 14 cidades acompanhadas, o OE2012 classificou nas primeiras posições (como usuários influentes) vários veículos de comunicação, que tradicionalmente sempre influenciaram o eleitorado. Por exemplo, em São Paulo temos nas primeiras colocações instituições como Folha, Estadão, Veja, Carta Capital e jornalistas como José Simão, Mônica Bergamo e Soninha Francine. Já no Rio de Janeiro temos O Globo, Veja, G1 e personalidades como Carlos Latuff, Jean Wyllys e Felipe Neto. Este último é um exemplo especialmente interessante, pois representa um caso de ascensão por meio das redes sociais na Web. Felipe Neto saiu do anonimato através de seus tweets irreverentes em 2010; hoje é apresentador e tem seu próprio programa em um canal de TV. Além dos veículos de comunicações, de jornalistas e personalidades, todos os rankings apresentaram perfis relacionados aos principais candidatos de suas respectivas cidades. Essas características apresentam fortes evidências da qualidade dos resultados proporcionados pela nossa métrica.

Dada a dinâmica das redes sociais na Web, era de se esperar ainda que perfis de usuários como os citados anteriormente estariam intercalados com usuários com pouca tradição e reconhecimento. E é interessante ressaltar que nossa técnica é capaz de capturar essa dinâmica, uma vez que ela identifica diversos perfis humorísticos como usuários influentes. $\mathrm{O}$ humor é um conteúdo que se propaga com facilidade nas redes sociais, e a política brasileira aparece frequentemente ligada ao humor, especialmente na internet. Charges, trocadilhos e piadas sobre candidatos invadem a Web e as redes sociais em época de eleições, ganhando grande repercussão entre os usuários.

Com exceção de São Paulo e do Rio de Janeiro, o ranking por cidade é formado por perfis mais "sérios". Os usuários influentes são, em geral, de veículos de comunicação, de cabos eleitorais e dos própios candidatos e/ou suas campanhas. Já no ranking global, é interessante notar que perfis humorísticos aparecem em grande escala, mais do que em cidades isoladas. Isso evidencia mais uma vez a capacidade da nossa técnica de capturar usuários influentes em cada contexto. Normalmente, usuários de uma cidade não estão interessados nos conteúdos relacionados às eleições em outras cidades, mas apenas nos relacionados à eleição em sua própria cidade. Quando se interessam por conteúdos relacionados às eleições de outras cidades, esses conteúdos são muitas vezes humorísticos. Dessa forma, os perfis hu- 
Uma Estratégia Baseada em Difusão de Informação para Determinação de Conteúdos Relevantes e Usuários Influentes em Redes Sociais.

morísticos que são secundários na esfera municipal, se tornam significativos nacionalmente.

Neste ponto, vale voltarmos à ideia originalmente tratada neste trabalho, que é relacionar a relevância do conteúdo à influência do usuário. Para veículos de comunicação já estabelecidos, como o jornal Folha por exemplo, praticamente qualquer publicação é facilmente propagada pela rede, pois é considerada uma informação vinda de uma fonte confiável. Este tipo de perfil é então facilmente considerado como influente. Já para um perfil anônimo, dificilmente ele conseguirá propagar na rede um conteúdo relevante que não seja humor, pois quem daria atenção à opinião política de um desconhecido? Entretanto, dado que diversos usuários não se interessam por política em si, mas facilmente consomem e propagam conteúdos humorísticos sobre as eleições e sobre os candidatos, tais perfis ganham importância, sendo capazes de produzir conteúdo relevante para vários usuários, e logo, tornando-se influentes.

\subsection{Casos de uso}

Nesta seção apresentamos alguns possíveis casos de uso que poderiam se beneficiar através da adoção da métrica proposta neste trabalho.

Ciências políticas e sociais são casos em que uma aplicação como essa poderia ajudar na produção de conhecimento. Por exemplo, uma das mais crescentes áreas da teoria política contemporânea e ciências sociais é o estudo da Democracia Deliberativa [14]. Nesse modelo democrático, enfatiza-se o uso da lógica e da razão, onde todos podem influenciar não apenas nas eleições, mas também nas decisões. Dessa forma, temos um cenário semelhante ao que podemos ver em redes sociais na Web.

Outra possibilidade de uso está no processo eleitoral. Candidatos costumam aderir às redes sociais para conhecer a opinião pública. Dados de pesquisas são importantes fontes de informação que mostram qual é a visão do eleitor sobre um candidato. Entretanto, tais dados relatam pouco mais do que sua popularidade. Uma ferramenta como a apresentada, que mostra de forma rica como as propostas estão sendo assimiladas pelos eleitores, pode contribuir para uma divulgação mais clara das propostas dos candidatos. Consequentemente, contribui para um processo eleitoral mais eficiente.

Além do cenário político, como o discutido neste estudo de caso, a métrica proposta pode ser implementada em qualquer cenário em que se deseja ter acesso a quem são os usuários responsáveis por formar uma opinião, e/ou influenciar uma grande quantidade de outros usuários, além de acompanhar o conteúdo propagado na rede considerado relevante sobre determinado assunto. Avaliar a repercussão de uma figura pública, uma empresa ou uma nova propaganda para divulgação de uma marca são apenas alguns exemplos de novos cenários plausíveis para a aplicação da nossa métrica. 
Uma Estratégia Baseada em Difusão de Informação para Determinação de Conteúdos Relevantes e Usuários Influentes em Redes Sociais.

\section{Conclusões}

Neste artigo, propusemos uma nova técnica para ordenar usuários e conteúdos, de acordo com a sua influência e sua relevância, respectivamente. Essa nova técnica se baseia em uma definição intuitiva e circular de relevância e influência, ou seja, usuários influentes tendem a disseminar conteúdos relevantes e conteúdos relevantes são em geral disseminados por usuários influentes. Nossa técnica foi avaliada utilizando duas bases de dados reais do Twitter. Os resultados obtidos mostram que a técnica proposta apresenta ganhos de $37 \%$ quando comparada a um método de filtragem colaborativa para recomendação. Nossa técnica aponta ainda como usuários influentes aqueles que realmente são influentes na rede (agências de notícias, humoristas e personagens famosos). Além disso, classificamos como relevantes conteúdos que são muito disseminados na rede, especialmente por usuários influentes. Esse tipo de resultado não é obtido com a utilização de técnicas mais simples (e.g. contagem do número de seguidores ou do número de retweets). Por último, promovemos um estudo de caso, no qual aplicamos o método proposto em um cenário real da Web. A análise mostrou que nossa técnica é capaz de reconhecer usuários influentes dentro de um dado contexto, e que pode ser facilmente adaptada e utilizada em várias aplicações. Em termos de trabalhos futuros, pretendemos construir um modelo formal, avaliar a tarefa de recomendação de usuários a seguir em redes sociais como o Twitter, além de comparar o nosso método com outros da literatura, assim como a nossa medida de influência com outras medidas já usadas na literatura. Todas essas tarefas devem considerar outras bases de dados reais.

\section{Agradecimentos}

Esse trabalho foi parcialmente financiado por CNPq, CAPES, Fapemig, e INWEB Instituto Nacional de Ciência e Tecnologia para a Web. Gostaríamos de agradecer também a toda a equipe responsável pelo Observatório da Web, especialmente a Walter dos Santos Filho.

\section{Referências}

[1] Sinan Aral, Lev Muchnik, and Arun Sundararajan. Distinguishing influence-based contagion from homophily-driven diffusion in dynamic networks. Proceedings of the $\mathrm{Na}$ tional Academy of Sciences, 2009.

[2] Sinan Aral and Dylan Walker. Identifying influential and susceptible members of social networks. Science, 337(6092):337-341, 2012.

[3] R. Baeza-Yates, B. Ribeiro-Neto, et al. Modern information retrieval, volume 82. Addison-Wesley New York, 1999. 
Uma Estratégia Baseada em Difusão de Informação para Determinação de Conteúdos Relevantes e Usuários Influentes em Redes Sociais.

[4] Shumeet Baluja, Rohan Seth, D. Sivakumar, Yushi Jing, Jay Yagnik, Shankar Kumar, Deepak Ravichandran, and Mohamed Aly. Video suggestion and discovery for youtube: taking random walks through the view graph. In Proceedings of the 17th international conference on World Wide Web, pages 895-904, New York, NY, USA, 2008. ACM.

[5] Sergey Brin and Lawrence Page. The anatomy of a large-scale hypertextual web search engine. Comput. Netw. ISDN Syst., 30(1-7):107-117, 1998.

[6] Meeyoung Cha, Hamed Haddadi, Fabricio Benevenuto, and Krishna P. Gummadi. Measuring User Influence in Twitter: The Million Follower Fallacy. In In Proceedings of the 4th International AAAI Conference on Weblogs and Social Media, Washington DC, USA, May 2010.

[7] Meeyoung Cha, Alan Mislove, and Krishna P. Gummadi. A measurement-driven analysis of information propagation in the flickr social network. In $W W W$ '09: Proceedings of the 18th international conference on World wide web, pages 721-730, New York, NY, USA, 2009. ACM.

[8] Massimo Franceschet. Pagerank: standing on the shoulders of giants. Commun. ACM, 54(6):92-101, June 2011.

[9] N.E. Friedkin. A structural theory of social influence, volume 13. Cambridge University Press, 2006.

[10] G. Frobenius. Über Matrizen aus nicht Negativen Elementen. 1912.

[11] Manuel Gomez Rodriguez, Jure Leskovec, and Andreas Krause. Inferring networks of diffusion and influence. In SIGKDD, 2010.

[12] John Hannon, Kevin McCarthy, and Barry Smyth. Finding useful users on twitter: twittomender the followee recommender. In Proc. of the 33rd European conf. on Advances in information retrieval, pages 784-787, Berlin, Heidelberg, 2011. Springer-Verlag.

[13] Jose L. Iribarren and Esteban Moro. Information diffusion epidemics in social networks. ArXiv e-prints, Jun 2007. http://arxiv.org/abs/0706.0641.

[14] Paul T Jaeger. Deliberative democracy and the conceptual foundations of electronic government. Government Information Quarterly, 22(4):702-719, 2005.

[15] E. Katz and F. Paul. Personal Influence: The Part Played by People in the Flow of Mass Communications. Transaction Publishers, 2005.

[16] David Kempe, Jon Kleinberg, and Éva Tardos. Maximizing the spread of influence through a social network. In Proceedings of the ninth ACM SIGKDD international conference on Knowledge discovery and data mining, KDD '03, pages 137-146, New York, NY, USA, 2003. ACM. 
Uma Estratégia Baseada em Difusão de Informação para Determinação de Conteúdos Relevantes e Usuários Influentes em Redes Sociais.

[17] Jon M. Kleinberg. Authoritative sources in a hyperlinked environment. In Proc. of the 9th annual ACM-SIAM symposium on Discrete algorithms, pages 668-677, Philadelphia, PA, USA, 1998. Society for Industrial and Applied Mathematics.

[18] Yehuda Koren and Robert M. Bell. Advances in collaborative filtering. In Recommender Systems Handbook, pages 145-186. 2011.

[19] Alex Leavitt, Evan Burchard, David Fisher, and Sam Gilbert. The Influentials: New Approaches for Analyzing Influence on Twitter. Webecology Project, September 2009.

[20] Jure Leskovec, Lada A. Adamic, and Bernardo A. Huberman. The dynamics of viral marketing. ACM Trans. Web, 1(1):5, 2007.

[21] Jure Leskovec, Mary Mcglohon, Christos Faloutsos, Natalie Glance, and Matthew Hurst. Patterns of cascading behavior in large blog graphs. In SDM '03: Proc. of the 7th SIAM SDM Int'l Conf. on Data Mining, pages 551-556, Philadelphia, PA, USA, 2007. SIAM.

[22] R. V. Mises and H. Pollaczek-Geiringer. Praktische Verfahren der Gleichungsauflösung. Zamm-zeitschrift Fur Angewandte Mathematik Und Mechanik, 9:58-77, 1929.

[23] Paul Resnick and Hal Varian. Recommender systems. Commun. ACM, 40(3):56-58, March 1997.

[24] Daniel M. Romero, Wojciech Galuba, Sitaram Asur, and Bernardo A. Huberman. Influence and passivity in social media. In Proceedings of the 20th international conference companion on World wide web, pages 113-114, New York, NY, USA, 2011.

[25] Arlei Silva, Hérico Valiati, Sara Guimarães, and Wagner Meira Jr. From individual behavior to influence networks: A case study on twitter. In Proc. of the 17th Brazilian Symposium on Multimedia, Hypermedia and Web, Porto Alegre, RS, Brazil, 2011. SBC.

[26] Hanghang Tong, Christos Faloutsos, and Jia-Yu Pan. Fast random walk with restart and its applications. In Proceedings of the Sixth International Conference on Data Mining, pages 613-622, Washington, DC, USA, 2006. IEEE Computer Society.

[27] Ibrahim Uysal and W. Bruce Croft. User oriented tweet ranking: a filtering approach to microblogs. In Proc. of the 20th ACM international conference on Information and knowledge management, pages 2261-2264, NY, NY, USA, 2011. ACM.

[28] Hérico Valiati, Arlei Silva, Sara Guimarães, and Wagner Meira Jr. Detecção de conteúdo relevante e usuários influentes no twitter. In Proc. of the Brazilian Workshop on Social Network Analysis and Mining (Brasnam), 2012. 
Uma Estratégia Baseada em Difusão de Informação para Determinação de Conteúdos Relevantes e Usuários Influentes em Redes Sociais.

[29] Jianshu Weng, Ee-Peng Lim, Jing Jiang, and Qi He. Twitterrank: finding topic-sensitive influential twitterers. In Proceedings of the 3rd ACM international conference on Web search and data mining, pages 261-270. ACM, 2010. 